
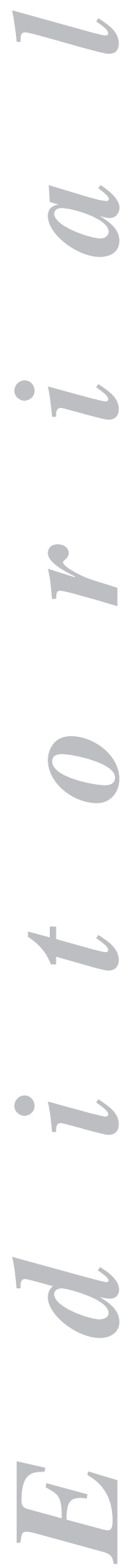

\title{
Dieta y cáncer de colon
}

El carcinoma colorrectal (CCR) es una de las neoplasias más prevalentes en los países occidentales y en nuestro país concretamente, es la segunda causa más frecuente de cáncer entre todos los tipos de tumores malignos, estando precedida únicamente por la localización en pulmón en varones y por la mama en la mujer. Su incidencia en España se estima en unos 25.000 casos diagnosticados por año, con una mortalidad de alrededor del 50\%, lo que representa que unos 12.500 pacientes fallecen anualmente por CCR en nuestro país, representando por tanto, la segunda causa de muerte por cáncer a nivel nacional (1) (Fig. 1).

Aunque la mayoría de los casos $(70-75 \%)$ corresponden a formas esporádicas, en las que por el momento se desconocen cuáles son los factores predisponentes para su desarrollo, en el 25-30\% restante puede existir una historia familiar que favorezca el desarrollo y aparición de esta neoplasia.

Así en el 3-5\% de los casos el CCR aparece en el contexto de una

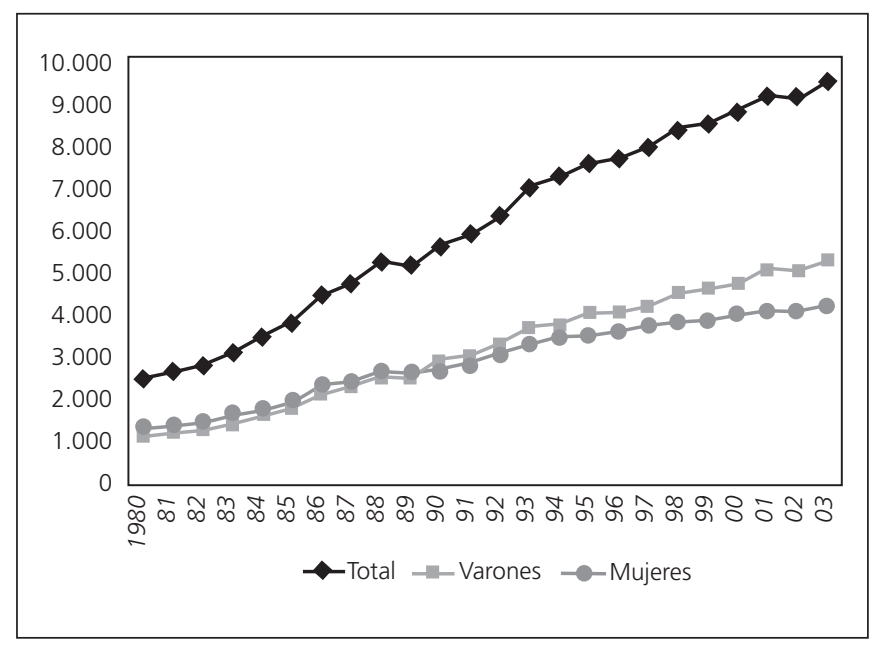

Fig. 1. Mortalidad observada por cáncer de colon en España (1980-2003). enfermedad con una base hereditaria conocida, fundamentalmente la poliposis adenomatosa familiar (PAF) cuya prevalencia es del 1\%, o en el seno de una forma de CCR hereditario no asociado a poliposis (CCHNP) o síndrome de Lynch, cuya prevalencia en nuestro país es del orden del 2,5\%, como ha sido elegantemente demostrado a través de un excelente estudio multicéntrico nacional, denominado Epicolon I, llevado a cabo con dicha finalidad por un grupo colaborativo dentro de la Asociación Española de Gastroenterología (2). Esta cifra es ligeramente inferior a la registrada en países americanos y europeos, con la excepción de Italia (3-5). En menos del 1\% el CCR aparece en pacientes con enfermedad inflamatoria intestinal (Fig. 2). 


\section{Relación entre pólipos de colon y desarrollo de CCR}

El término "pólipo" comprende cualquier crecimiento circunscrito que hace protrusión desde la pared hacia la luz intestinal. Dependiendo de su naturaleza histoló-

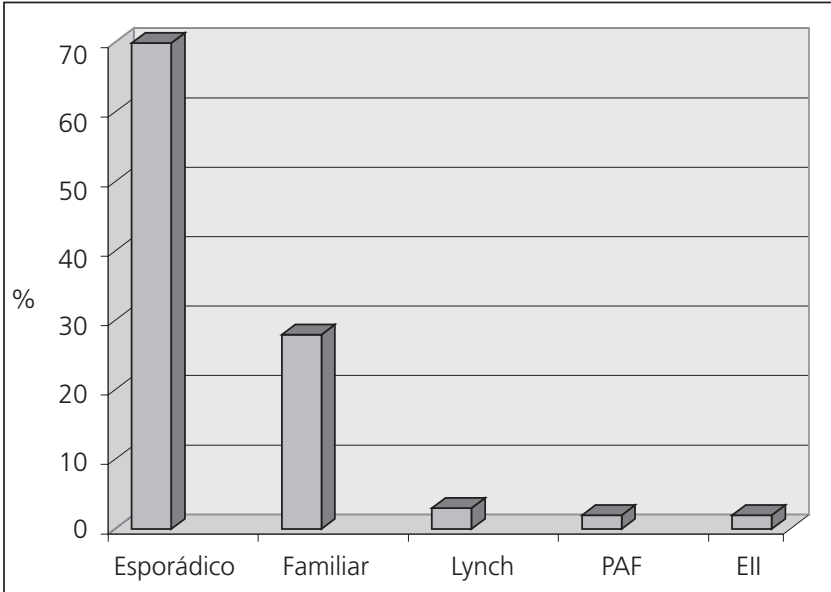

Fig. 2. Distribución de los distintos tipos de CCR. PAF: poliposis adenomatosa familiar; Ell: enfermedad inflamatoria intestinal. gica, los pólipos colorrectales se clasifican en neoplásicos y no neoplásicos.

Los pólipos neoplásicos del colon incluyen fundamentalmente a los adenomas (tubulares, túbulo-vellosos y vellosos) que representan entre el 70-80\% de todos los pólipos colorrectales, mientras que los restantes no neoplásicos comprenden los hiperplásicos, serrados, juveniles, inflamatorios $\mathrm{y}$ hamartomatosos.

Los adenomas son las lesiones precursoras de la mayoría de los CCR detectados en el mundo occidental. Actualmente se acepta que la gran mayoría de CCR se producen como consecuencia de alteraciones genéticas que conducen a la transformación del epitelio colónico, primero en adenoma y finalmente en adenocarcinoma. La poliposis adenomatosa familiar (PAF) es una enfermedad hereditaria, que se transmite con carácter autosómico dominante, y que está causada por mutaciones heredadas en el gen APC (adenomatous polyposis coli). Su frecuencia es baja y representa aproximadamente el $1 \%$ de todos los casos de CCR, caracterizándose por la aparición de múltiples pólipos adenomatosos del colon (más de 100), en edades tempranas de la vida, y con un riesgo de desarrollar CCR del $100 \%$ (6). El conocimiento de los mecanismos genéticos que llevan al desarrollo de CCR en la PAF ha permitido conocer mejor cuáles son las etapas en el proceso de carcinogénesis colónica. El primer paso consiste en la inactivación del gen supresor APC, sumándose posteriormente la activación del oncogen $\mathrm{K}$ Ras y la inactivación de otros genes supresores (TP53, SMAD4 y otros); todo ello conduce al desarrollo del carcinoma colorrectal (Fig. 3 ).

Además del anterior proceso de carcinogénesis, Fig. 3. 
existe otra vía alternativa que consiste en la acumulación de mutaciones en fragmentos repetitivos del ADN (microsatélites), como consecuencia de la alteración de los mecanismos responsables de la reparación de los errores de replicación del ADN. Esta vía, denominada mutadora, tiene lugar principalmente en los pacientes portadores de un carcinoma hereditario no polipoideo (CCRHNP) (7).

El 20-40\% de la población asintomática mayor de 50 años, presenta adenomas colorrectales. Sin embargo, aproximadamente el $80 \%$ presenta sólo uno o dos pólipos y estos son de pequeño tamaño $(<1 \mathrm{~cm})$, con un bajo potencial de malignización. Los adenomas que presentan un alto riesgo de poder convertirse en CCR, son aquellos que tienen un tamaño superior a $1 \mathrm{~cm}$, presentan displasia grave de alto grado (carcinoma in situ) o carcinoma invasor, por lo que se les denomina genéricamente como adenomas avanzados. Aproximadamente el 5\% de los adenomas colorrectales presentan transformación carcinomatosa (8). El crecimiento de estas lesiones es lento, de forma que un pólipo menor de $1 \mathrm{~cm}$, tarda aproximadamente entre 5-10 años en malignizarse (9).

La evidencia abrumadora existente en la actualidad sobre el importante papel que desempeñan los pólipos adenomatosos del colon en la carcinogénesis colónica, señala bien a las claras que las estrategias de prevención del CCR, estén dirigidas preferentemente a la detección precoz de estas lesiones.

\section{Prevención primaria del CCR}

Además del diagnóstico precoz de las lesiones precursoras del CCR (adenomas), existen otras estrategias que pretenden disminuir el riesgo de desarrollo de CCR (prevención primaria), como son la quimioprofilaxis con AAS o COXIB (inhibidores de la $\mathrm{COX}-2$ ) y las modificaciones en los hábitos de vida y dietéticos; esta última aproximación se fundamenta en la existencia de factores ambientales en el CCR. Existen grandes diferencias geográficas en la incidencia y mortalidad de este tipo de cáncer entre diversos países y razas a nivel mundial, observándose un incremento progresivo en el número de casos en diversos lugares y registrándose un evidente aumento en la incidencia entre los emigrantes procedentes de áreas de bajo riesgo, cuando se trasladan a vivir a otros países de riesgo más elevado, lo que habla claramente a favor de la gran influencia que desempeñan diversos factores ambientales en su presentación y desarrollo.

\section{Influencia del estilo de vida: consumo de tabaco y alcohol, actividad física y obesidad}

Las diferencias existentes en los estilos de vida de las diversas poblaciones a nivel mundial pudieran desempeñar un papel importante en el riesgo de desarrollo de CCR; así el consumo de tabaco y alcohol, la falta de actividad física y la obesidad, constituyen por sí solos y también asociados, factores de riesgo para el desarrollo del CCR (10). La exposición a los productos derivados del tabaco de forma temprana, se asocia con un riesgo aumentado para el desarrollo del carcinoma colorrectal.

Así por ejemplo, es bien conocido que el consumo excesivo de alcohol es un factor de riesgo, tanto en hombre como en mujeres, para el desarrollo de cáncer de colon y de recto. Un reciente análisis conjunto de 8 estudios de cohorte, que incluía medio millón de personas de 5 países distintos (11), mostró que el riesgo es- 
taba aumentado a partir de un consumo de 30 gramos de alcohol por día, y era independiente del tipo de bebida consumida.

La relación entre actividad física y CCR no está bien aclarada, pues si bien los estudios caso-control encuentran un efecto protector, no ocurre lo mismo con los estudios de cohortes; por otra parte, la actividad física no parece influir en el desarrollo de cáncer de recto (12). Diversos estudios epidemiológicos han puesto en evidencia la existencia de una relación clara entre sobrepeso y obesidad y el riesgo de cáncer de colon (no de recto) en hombres (no en mujeres) (13), y esta asociación es más pronunciada en personas con escasa actividad física.

El fenómeno de "resistencia a la insulina" parece que puede jugar un cierto papel, pues la exposición del colonocito a concentraciones elevadas de insulina induce un efecto mitogénico en estas células, mientras que la exposición a la glucosa y ácidos grasos se relaciona con alteraciones metabólicas y aumento del estrés oxidativo (14). Por otra parte, los pacientes obesos presentan un riesgo aumentado para desarrollar complicaciones tras cirugía abdominal, incluyendo infección y dehiscencia de suturas, presencia de linfedema, aparición de otros cánceres y complicaciones relacionadas con las enfermedades crónicas que se asocian frecuentemente con los pacientes obesos sin cáncer, tales como las enfermedades cardiovasculares y la diabetes. Los mecanismos propuestos para explicar la asociación entre obesidad y peor pronóstico (15), incluyen un incremento en el tejido adiposo de las concentraciones de estrógenos y testosterona, insulina, factores de crecimiento relacionados con la insulina (IGF), leptina y diversas citoquinas. Como mecanismos adicionales se postula una disminución en la función immune en general, una peor dosificación de los fármacos empleados (especialmente los quimioterápicos) y diferencias en la actividad física y accidentes trombóticos y cardiovasculares en pacientes con sobrepeso, comparados con controles.

Es interesante recordar que la diabetes mellitus y el CCR comparten varios factores de riesgo, habiéndose demostrado que los niveles de péptido $\mathrm{C}$ en plasma constituyen por sí solos, un factor predictivo de aparición y desarrollo del CCR, independiente del índice de masa corporal (16).

\section{Frutas, vegetales y fibra}

Aunque hasta hace pocos años, relativamente, se había asumido a partir de estudios caso-control que la ingesta de fruta y vegetales disminuiría el riesgo de CCR, los estudios de cohortes han mostrado resultados discordantes (efecto beneficioso en unos y ningún efecto en otros). Tomados en conjunto, el consumo de estos alimentos podría tener, quizás, un pequeño papel preventivo en el desarrollo del CCR (17).

Se ha relacionado la ingesta de fibra con una disminución del riesgo de CCR en base a evidencias epidemiológicas (menor incidencia de CCR en sociedades subdesarrolladas en las que hay una importante ingesta de fibra) y a teorías patogénicas (tránsito intestinal acelerado que impediría el contacto de los carcinógenos con la mucosa del colon). Estudios caso-control mostraron un efecto protector de la fibra, mientras que un reciente análisis conjunto de los estudios de cohorte publicados, que incluyó más de 8.000 casos, no pudo demostrar este efecto beneficioso (18). Una explicación de los distintos efectos publicados podría ser que aunque la cantidad de fibra sea la misma en las cohortes analizadas, la composición de la misma puede ser muy diferente (fibra soluble o no soluble, de origen en cereales, 
frutas o vegetales). Por otra parte, los suplementos de fibra no han sido eficaces en la prevención de la recurrencia de los adenomas de colon (19).

\section{Relación con el consumo de carne roja y grasa}

Al igual que ocurría con los anteriores nutrientes, los estudios sobre la relación entre consumo de carne roja (ovino, bovino y porcino) y CCR muestran resultados dispares, probablemente por la inclusión de carnes procesadas o manufacturadas (por ejemplo, salchichas) en algunos de ellos, y por la distinta forma de preparación de las carnes. Los estudios más recientes con cohortes amplias sí muestran una asociación débil entre consumo de carne roja y CCR (20).

Se cree que los distintos métodos de preparación de la carne roja (frita, parrilla) van a generar distintas sustancias mutagénicas (aminas heterocíclicas, hidrocarbonos aromáticos policíclicos) dentro de la carne; por otra parte, es posible que personas con un genotipo acetilador rápido sean más susceptibles a estos carcinógenos de la carne.

Parece existir una asociación clara entre consumo de carnes procesadas y riesgo de CCR, que se ha llegado a cuantificar en un incremento del 50\% para una ingesta diaria de $25 \mathrm{~g}$ de este tipo de carne (20). Se especula que este riesgo es debido a la presencia de nitrosaminas en la carne curada.

La ingesta de grasa no se ha podido relacionar con un mayor riesgo de CCR.

\section{Lácteos y calcio}

El consumo de productos lácteos se ha asociado en un análisis conjunto de 10 estudios de cohorte que incluían 5.000 casos, con una disminución de un 11\% del riesgo de CCR (21). Esta asociación se ha visto también con los suplementos de calcio, que disminuyen en un $19 \%$ la recurrencia de adenomas de colon respecto a un grupo con placebo (22).

\section{Comentarios al trabajo español actual publicado sobre el tema}

En el presente número de la revista, Solera Albero y cols. presentan los resultados de un estudio poblacional, transversal, a nivel de Atención Primaria en tres zonas de la provincia de Albacete, sobre la influencia de ciertos hábitos de vida y dietéticos en el riesgo de desarrollar CCR (23). Realizaron una encuesta a través de un cuestionario escrito en 414 individuos diagnosticados de cáncer colorrectal, mayores de 50 años, de los que $55 \%$ eran varones y $45 \%$ mujeres, con una análisis estadístico multivariante y de regresión logística, en el que analizan diversas variables tanto de estilo de vida, como del tipo de alimentación. Obtienen una correlación significativa con distintas variables esperadas, tales como la presencia de antecedentes familiares de primer grado, un cierto grado de sedentarismo y un moderado o elevado consumo de alcohol.

Curiosamente los autores encuentran una relación directa con el consumo diario de agua y por encima de los 2 litros al día, observan una mayor incidencia de CCR.

Asimismo observan una cierta correlación con el consumo de pasta, arroz y huevos. Hubiese sido interesante separar el consumo de pasta (hecha con cereales que contienen gluten) del arroz (por no contener gluten) y resulta curiosa también la 
asociación encontrada con el consumo de huevos, que resulta de difícil explicación.

$\mathrm{Al}$ igual que otros estudios internacionales no encuentran una clara relación con el consumo de fibra vegetal, carne roja o pescados y es interesante el probable efecto beneficioso observado con el consumo de aceite de oliva.

Es de resaltar la gran dificultad existente en la realización de este tipo de estudios epidemiológicos, basados en encuestas poblacionales en grupos de riesgo, máxime con el amplio abanico de cuestiones planteadas y la variabilidad y riqueza de la dieta española. Ello hace que el intentar establecer correlaciones de causalidad, sea extremadamente difícil, por el importante sesgo que supone de por sí, el contestar con rigor y fiabilidad a las diferentes preguntas que la componen.

Ello, no obstante, no resta ni un ápice de mérito e interés a la calidad del trabajo realizado y al gran esfuerzo llevado a cabo, en el diseño, ejecución e interpretación de los resultados obtenidos. Es justo decir que se trata del primer trabajo llevado a cabo en España sobre este interesante tema y que debería servir de ejemplo y estímulo para la realización de otros posteriores en diversas regiones de nuestro país.

Existe una enorme falta de interés en nuestro medio por este tipo de trabajos epidemiológicos y una auténtica sequía de publicaciones realizadas sobre este tema en lengua española. Así en una búsqueda reciente llevada a cabo en el buscador bibliográfico médico internacional más utilizado PUBMED, bajo los indicadores de "Dieta y cáncer de colon", sólo hemos podido encontrar 6 publicaciones en español, frente a un total de 2.483 realizadas en inglés.

Curiosamente la última publicación española fue realizada hace veinte años por el grupo del Dr. Pajares del Servicio de Digestivo del Hospital de La Princesa de Madrid, en el que en un estudio retrospectivo describieron los cambios observados en la frecuencia del cáncer de colon y gástrico en su hospital, en un periodo de 20 años (1960-79) en el que ya se comenzaba a ver un descenso progresivo en la incidencia del cáncer de estómago asociado con un aumento correspondiente del cáncer de colon, con un cruce en sus rectas (una descendente y otra descendente) que se mantienen mucho más claramente hasta la actualidad (24).

Hay que recordar también que existe una excelente revisión sobre este tema publicada recientemente, hace dos años, en las que se analizan de forma detallada los diversos factores dietéticos posiblemente implicados (25).

Aunque existen muchos mecanismos por aclarar, existe una cierta evidencia procedente de diversos estudios epidemiológicos y experimentales, que diversos factores dietéticos, ambientales y relacionados con el estilo de vida tienen una gran influencia en el desarrollo del CCR.

De los micronutrientes y vitaminas analizados, el que presenta un mejor perfil protector por el momento es el ácido fólico y su efecto beneficioso está siendo estudiado en diversos estudios controlados que se están llevando a cabo en la actualidad.

Confiemos que la publicación de este trabajo, sirva de acicate a nuevos grupos de trabajo para al realización de diversos estudios a nivel nacional, que intenten encontrar correlaciones interesantes entre la dieta y el CCR (no olvidemos que el 70\% de los casos son esporádicos) y que incluso pudieran demostrar el posible efecto beneficioso de la dieta mediterránea en su prevención. 


\section{Bibliografía}

1. Castells A, Marzo M, Bellas B, Amador F, Lanas A, Mascort J, et al. Guía de práctica clínica en prevención del cáncer colorrectal. Gastroenterol Hepatol 2004; 27: 573-634.

2. Piñol V, Andreu M, Castells A, Payá A, Bessa X, Jover R. Frequency of hereditary non-polyposis colorectal cancer and other colorectal cancer familial forms in Spain. A multicenter, prospective, nation-wide study. Gastrointestinal Oncology Group of the Spanish Gastroenterological Association. Eur J Gastroenterol Hepatol 2004; 16: 39-45.

3. Hampel H, Frankel WL, Martin E, Arnold M, Khanduja K, Kuebler P, et al. Screening for the Lynch syndrome (hereditary nonpolyposis colorectal cancer). N Engl J Med 2005; 352: 1851-60.

4. Lindor NM, Burgart LJ, Leontovich O, Goldberg RM, Cunningham JM, Sargent DJ, et al. Immunohistochemistry versus microsatellite instability testing in phenotyping colorectal tumors. J Clin Oncol 2002; 20: 10438.

5. Percesepe A, Borghi F, Menigatti M, Losi L, Foroni M, Di Gregorio C, et al. Molecular screening for hereditary nonpolyposis colorectal cancer: A prospective, population-based study. J Clin Oncol 2001; 19: 3944-50.

6. Pares D, Pera M, González S, Pascual Cruz M, Blanco I. Poliposis adenomatosa familiar. Gastroenterol Hepatol 2006; 29: 625-35.

7. Lynch HT, De la Chapelle A. Hereditary colorectal cancer. N Engl J Med 2003; 348: 919-32.

8. Anwar S, White J, Hall C, Farrell WE, Deakin M, Elder JB. Sporadic colorectal polyps: Management options and guidelines. Scand J Gastroenterol 1999; 34: 4-11.

9. Atkin WS, Saunders BP. Surveillance guidelines after removal of colorectal adenomatous polyps. Gut 2002; 51: V6-V9.

10. Martinez ME. Primary prevention of colorectal cancer: Lifestyle, nutrition, exercise. Recent Results Cancer Res 2005; 166: 177-211.

11. Cho E, Smith-Warner SA, Ritz J, Van den Brandt PA, Colditz GA, Folsom AR, et al. Alcohol intake and colorectal cancer: A pooled analysis of 8 cohort studies. Ann Intern Med 2004; 140: 603-13.

12. Van den Brandt PA, Goldbohm RA. Nutrition in the prevention of gastrointestinal cancer. Best Pract Res Clin Gastroenterol 2006; 20: 589-603.

13. Bergstrom A, Pisani P, Tenet V, Wolk A, Adami HO. Overweight as an avoidable cause of cancer in Europe. Int J Cancer 2001; 91: 421-30.

14. Gunter MJ, Leitzmann MF. Obesity and colorectal cancer: Epidemiology, mechanisms and candidate genes. J Nutr Biochem 2006; 17: 145-56.

15. McTiernan A. Obesity and cancer: The risks, science, and potential management strategies. Oncology 2005; 19: 871-81.

16. Ma J, Giovannucci E, Pollak M, Leavitt A, Tao Y, Gaziano JM, et al. A prospective study of plasma C-peptide and colorectal cancer risk in men. J Natl Cancer Inst 2004; 96: 546-53.

17. Park Y, Hunter DJ, Spiegelman D, Bergkvist L, Berrino F, Van den Brandt PA, et al. Dietary fiber intake and risk of colorectal cancer: A pooled analysis of prospective cohort studies. JAMA 2005; 294: 2849-57.

18. Alberts DS, Martínez ME, Roe DJ, Guillén-Rodríguez JM, Marshall JR, Van Leeuwen JB, et al. Lack of effect of a high-fiber cereal supplement on the recurrence of colorectal adenomas. Phoenix Colon Cancer Prevention Physician's Network. N Engl J Med 2000; 342: 1156-62.

19. Norat T, Bingham S, Ferrari P, Slimani N, Jenab M, Mazuir M, et al. Meat, fish and colorectal cancer risk: The European Prospective Investigation into Cancer and Nutrition. J Natl Cancer Inst 2005; 97: 906-16.

20. Sandhu MS, White IR, McPherson K. Systematic review of the prospective cohort studies on meat consumption and colorectal cancer risk: A meta-analytical approach. Cancer Epidemiol Biomarkers Prev 2001; 10: 439-46.

21. Cho E, Smith-Warner SA, Spiegelman D, Beeson WL, Van den Brandt PA, Colditz GA, et al. Dairy foods, calcium and colorectal cancer: A pooled analysis of 10 cohort studies. J Natl Cancer Inst 2004; 96: 1015-22.

22. Baron JA, Beach M, Mandel JS, Van Stolk RU, Haile RW, Sandler RS, et al. Calcium supplements for the prevention of colorectal adenomas. Calcium Polyp Prevention Study Group. N Engl J Med 1999; 340: 101-7.

23. Solera Albero J, Tárraga López PJ, López Cara MA, Celada Rodríguez A, Cerdán Oliver M, Ocaña López JM. Influencia de la dieta y los estilos de vida en el cáncer colorrectal. Rev Esp Enferm Dig 2007; 99: 190200.

24. Maté Jiménez J, Rodríguez Muñoz S, Mearin F, Pajares JM. Frecuencia del carcinoma de colon y de estómago en el período 1960-1979 en relación con los cambios dieteticos y migratorios. Rev Esp Enferm Dig 1987; 71: 31-7.

25. Franco A, Sikalidis AK, Solís Herruzo JA. Colorectal cancer: influence of diet and lifestyle factors. Rev Esp Enferm Dig 2005; 97: 432-48. 